\title{
Considerations for the Clinical Use of Evidence- Based Practice Approaches for Co-Occurring Disorders
}

ISSN: 2578-0042

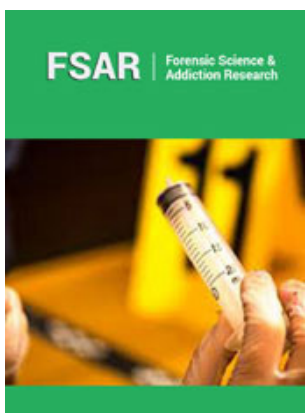

*Corresponding author: Michael J Tkach, Department of Psychology, USA

Submission: 侮-1 May 02, 2019

Published: 䟧 May 17, 2019

Volume 4 - Issue 4

How to cite this article: Michael Tkach $J^{*}$, Considerations for the Clinical Use of Evidence-Based Practice Approaches for Co-Occurring Disorders. Forensic Sci Add Res. 4(4). FSAR.000600.2019.

DOI: 10.31031/FSAR.2019.04.000600

Copyright@ Michael Tkach J, This article is distributed under the terms of the Creative Commons Attribution 4.0 International License, which permits unrestricted use and redistribution provided that the original author and source are credited.
Michael J Tkach*

Adjunct Professor of Hazelden Betty Ford, USA

\section{Abstract}

The use of Evidence-Based Practice approaches has become the industry standard for the treatment of co-occurring disorders; however, there are many factors for clinicians to consider when selecting and adapting these approaches for effective clinical use. The author of this article reviews briefly the history of the Evidence-Based Practice movement and how both substance use treatment and mental health field approached this topic. Finally, recommendations are made suggesting that cultural adaptations of evidence-based practice approaches should consider the following four factors:

1. The culture of the client

2. The clinician's culture and competency

3. The culture of the theory being used and

4. The culture of the treatment setting

Keywords: Evidence-based practice; Substance use treatment; Co-Occurring disorders; Empirically supported treatment; Cultural adaptations

\section{Introduction}

According to the 2017 National Survey of Substance Abuse Treatment Services, it is estimated that $37 \%$ of the individuals seeking treatment for substance use disorders had a co-occurring mental health disorder [1]. Atkins [2] estimated that more than 8 million Americans qualified for having a co-occurring disorder and reviewed historical approaches to the approach to treatment taken by the field noting that there has been shifts in perspective between recommending treating mental health and substance use disorder condition separately, sequentially, or simultaneously. The current trend in treatment is towards care integration and the treatment of co-occurring disorders simultaneously unless otherwise indicated by specific client needs.

Although the concurrent treatment of co-occurring disorders is often perceived as the best practice, many sites are not designed to provide truly co-occurring services and instead tend to have more proficiency towards providing either substance use or mental health treatment. For sites that are looking to provide co-occurring disorder treatment, clinicians are then faced with the challenge of selecting appropriate interventions and treatment modalities. With ethical practice guidelines aimed towards the use modalities that are established as efficacious there can be numerous reasons why a clinician would prefer to use evidencebased practices approaches as best practice [3]. Although the use of evidence-based practice approaches may be preferred and are often taught to new clinicians as the gold standard of treatment, there still seems to be a bit of confusion about what constitutes an evidence-based practice and how clinicians can best apply these approaches in clinical practice.

\section{Brief History of EBP}

According to the American Psychological Association [3], the heightened interest in the use of evidence-based practice (EBP) approaches started in 1999 in response to questions about the value of therapeutic interventions in comparison to (or in conjunction with) the use of psychotropic medications. The APA (2006) stated, "Evidence-based practice in psychology (EBPP) is the integration of the best available research with clinical expertise in the context of 
patient characteristics, culture, and preferences" (p. 273). Guidelines were established for how a practice goes from being an approach supported by research to being referred to as an established EBP. This included rigorous research protocols supporting efficacy conducted in a variety of ways (e.g., qualitative research, processoutcome studies, randomized control studies, meta-analyses, etc.). The APA was great at identifying that the research support helped an approach become an Empirically Supported Treatment (EST) but that it was the combination of an EST and clinical expertise that allows an approach to become an EBP.

Numerous studies have been published in the years since to help guide clinicians with the selection of EST's to use in providing EBP approaches. The common theme tends to be summarized in that the selection should be based on

\section{Efficiency; and}

\section{Clinical utility $[4,5]$.}

In terms of efficacy, clinicians are to research how effective the approach is in treating what the approach is used to treat, while clinical utility considers how robust the research is supporting its use as well as whether its use is supported for the treatment of the client. This may include several factors including supports for use with individuals from the client's culture, as well as appropriate for the treatment setting it is being used in [4]. The emphasis than shifts from strict fidelity and a one-size fits all approach to instead focusing on individualized treatment informed by the key factors that make that intervention effective $[3,4,6]$.

The complications for establishing effective EBP approaches for co-occurring disorders is related to the differences between the process for naming treatments of mental health and those of substance use disorders as EBP. The difference, as noted by SAMHSA [7] has resulted in a move to remove the National Registry of Evidence Based Programs and Practices from its website due to times where the standard was limited to only requiring two separate research articles supporting efficacy. That being stated, many of the approaches on the register were well substantiated in effectiveness; however, there is nevertheless a difference between the process used to establish mental health and substance use interventions as EBP. As such, often the approaches used in the literature to establish an approach as an EST to become an EBP for co-occurring disorder tends to repeat the pattern of favoring providing treatment towards either mental health or substance use symptoms, which can then further complicate selecting an EST to use when providing true co-occurring treatment.

\section{Selection Process Recommendations}

In order to help guide clinicians in the selection of EST's to apply as EBP approaches, it is recommended that clinicians consider multiple domains [8]. The domains explored often include considering the culture of the client and the culture of the clinician, while two additional considerations are also recommended: the culture of the theory in which the treatment was developed, and the culture of the treatment setting.

\section{Client considerations}

Proper selection of an EST to use as an EBP requires considering client characteristics to ensure that the research supports the use of an intervention that matches the unique needs of the client. This process includes ensuring that the EST was validated for use with the client's culture and population demographics as well as meets additional needs such as it is aligned with client preferences and affordability. Although many articles recognize the need to adapt EBP approaches to be treat clients, specifically when the client does not identify the with dominant culture, there are nevertheless still few research articles that outline how clinicians should actually process adapting an approach [5]. Additionally, the client's own self-identity even within the context of a larger cultural grouping may further determine whether the research supporting use is generalizable to that specific individual [9]. Fortunately, Chu et al. [10] reported on a review of research that supported that cultural adaptations of treatment approaches that are used in clinical practice are often effective [10]. They further suggested that the successful adaptation of approaches to fit the client's culture are often more effective when three circumstances are achieved:

1. 'A contextual match with clients' external realities;

2. An experiential match in the microsystem of the therapeutic relationship or framework; and

3. An intrapersonal feeling of being understood and empowerment within the client" [10].

\section{Clinician considerations}

In order to change a theoretical approach to best fit the needs of the client, an understanding of theory and research is required. By understanding the theory and research supporting an EST can help the clinician best be determined whether modifying the approach can be done in a way that matches the cultural needs of the individual being treated while still also maintaining the integrity of the factors of change that are deemed to be efficacious [5].

In terms of clinician awareness, the clinician is tasked with not only understanding their own cultural influences that may contribute to how they approach and interact with clients, but also to understanding how modifying approaches may stretch out of their areas of competency [8]. These cultural blind spots and lack of feeling comfortable with practicing in this manner is hypothesized to contribute to clinician resistance to adapt EBP approaches for the cultural needs of the client [9]. To best understand how to adapt an EST for a specific client population, an understanding of the theory used to construct the EST is important.

\section{Theory considerations}

Although likely overlooked and taken for granted by many dominant culture clinicians, the theories used in clinical practice that inform treatment approaches are created based on achieving cultural ideals. As Nagayama Hall et al. [6] noted, theoretical-based approaches are often designed in a way that supports the values and ideas of health of the culture within which these approaches 
are created and therefore provide a vehicle for supporting those cultural values through the use of that intervention. As such, the intervention itself may become the vehicle by which those cultural values are placed over the values of the individual that the approach is meant to support. Nagayama Hall and colleagues used the example of the inherit bias towards individualism and rational cognition that they identified within the theory supporting the use of Cognitive Behavioral Therapy (CBT), and how these values may not match with a more collectivist culture or one that places great value on emotions over rational cognitions. As such, clinicians are encouraged to understand the influence the culture in which an approach was created may have in its use with clients.

\section{Culture of the treatment setting}

Clinicians are also encouraged to consider the culture of the treatment setting itself when selecting EST's to use as EBP approaches. This may include identifying whether the treatment settings that the clinician practices in is primarily focused on either substance use treatment or mental health treatment [2]. Furthermore, clinicians are encouraged to consider how the perception of the treatment setting may influence beliefs and expectations of the client especially if the client is at different levels of preparedness to address what they see as separate presenting concerns. For example, a client presenting to a place known primarily for substance use disorder treatment might not otherwise be prepared for engaging in treatment of trauma. Although treatment of both are likely to benefit the client, the client may otherwise feel pressure to engage in treatment for trauma even if they are otherwise not ready if they were not prepared for the possibility of addressing both prior to engaging in services.

\section{Conclusion}

The idea of treating co-occurring disorders with EBP approaches may be easier theoretically than in practice. Many factors contribute to obstacles faced by clinicians when selecting and using EBP approaches effectively, including historical differences in the establishment of EBP for psychology and substance use treatment [3]. Clinicians seeking to select EST to become EBP approaches are encouraged to consider the following when adapting EST's to fit the client's needs and to be considered as an EBP:
1. Client's clinical needs and culture;

2. The clinician's culture and competency;

3. The culture of the theory being used; and

The culture of the treatment setting. By addressing these areas critically, clinicians can determine whether the factors believed to be effective in the use of an EBP approach would be appropriate for adaptation and use with a specific client.

\section{References}

1. National Survey of Substance Abuse Treatment Services (N-SSATS) (2017) Substance abuse and mental health services administration, Rockville, Maryland, USA.

2. Atkins C (2014) Co-occurring disorders: Integrated assessment and treatment of substance use and mental disorders. PESI Publishing, Eau Claire WI, USA.

3. APA Presidential Task Force on Evidence-Based Practice (2006) Evidence-based practice in psychology. Am Psychol 61(4): 271-285.

4. Valenzuaela JM, Pulgaron ER, Salamon KS, Patino-Fernandez AM (2017) Evidence-based assessment strategies for working with ethnic minority youth. Clinical Practice in Pediatric Psychology 5(1): 108-120.

5. Moore LA, Aarons GA, Davis JH, Novins DK (2015) How do providers serving American Indians and Alaska Natives with substance abuse problems define evidence-based treatment? Psychol Serv 12(2): 92-100.

6. Nagayama GC, Hall, Kim-Mozeleski, Jin E, Nolan W, et al. (2019) Cultural adaptations of psychotherapy: therapists' applications of conceptual models with Asians and Asian Americans. American Psychological Association 10(1): 68-78.

7. SAMHSA (2018) Statement of Elinore F McCance-Katz, MD, PhD, assistant secretary for mental health and substance use regarding the national registry of evidence-based programs and practices and asmhsa's new approach to implementation of evidence-based practices (EBP's), Rockville, Maryland, USA.

8. Domenech R, Melanie M, Baumann AA, Vázquez AL, Amador-Buenabad NG, et al. (2018) Scaling out evidence-based interventions outside the US mainland: Social justice or Trojan horse? Journal of Latina/o Psychology 6(4): 329-344.

9. DeFife, Drill J, Beinashowitz R, Ballantyne J, Plant L, etal. (2015) Practicebased psychotherapy research in a public health setting: Obstacles and opportunities. Journal of Psychotherapy Integration 25(4): 299-312.

10. Chu J, Leino A, Pflum S, Sue S (2016) A model for the theoretical basis of cultural competency to guide psychotherapy. Professional Psychology: Research and Practice 47(1): 18-29. 